\title{
Evidence of a SiO collimated outflow from a massive YSO in IRAS 17233-3606
}

\author{
S. Leurini ${ }^{1}$, C. Codella ${ }^{2}$, A. Gusdorf ${ }^{3}$, L. Zapata ${ }^{4}$, A. Gómez-Ruiz ${ }^{2}$, L. Testi ${ }^{5,2}$, and T. Pillai ${ }^{6}$ \\ 1 Max-Planck-Institut für Radioastronomie, Auf dem Hügel 69, 53121 Bonn, Germany \\ e-mail: sleurini@mpifr. de \\ 2 INAF - Osservatorio Astrofisico di Arcetri, Largo E. Fermi 5, 50125 Firenze, Italy \\ 3 LERMA, UMR 8112 du CNRS, Observatoire de Paris, École Normale Supérieure, 24 rue Lhomond, 75231 Paris Cedex 05, France \\ ${ }^{4}$ Centro de Radioastronomía y Astrofísica, Universidad Nacional Autónoma de México, 58090 Morelia, Mexico \\ 5 ESO, Karl-Schwarzschild Strasse 2, 85748 Garching-bei-München, Germany \\ ${ }^{6}$ Caltech, 1200 E. California Blvd, Pasadena, CA 91125, USA
}

Received 26 September 2011 / Accepted 3 April 2013

\begin{abstract}
Studies of molecular outflows in high-mass young stellar objects reveal important information about the formation process of massive stars. We therefore selected the close-by IRAS 17233-3606 massive star-forming region to perform SiO observations with the SMA interferometer in the (5-4) line and with the APEX single-dish telescope in the (5-4) and (8-7) transitions. In this paper, we present a study of one of the outflows in the region, OF1, which shows several properties similar to jets driven by low-mass protostars, such as $\mathrm{HH} 211$ and $\mathrm{HH} 212$. It is compact and collimated, and associated with extremely high velocity $\mathrm{CO}$ emission, and $\mathrm{SiO}$ emission at high velocities. We used a state-of-the-art shock model to constrain the pre-shock density and shock velocity of OF1. The model also allowed us to self-consistently estimate the mass of the OF1 outflow. The shock parameters inferred by the SiO modelling are comparable with those found for low-mass protostars, only with higher pre-shock density values, yielding an outflow mass in agreement with those obtained for molecular outflows driven by early B-type young stellar objects. Our study shows that it is possible to model the $\mathrm{SiO}$ emission in high-mass star-forming regions in the same way as for shocks from low-mass young stellar objects.
\end{abstract}

Key words. stars: protostars - stars: formation - ISM: jets and outflows - ISM: individual objects: IRAS 17233-3606

\section{Introduction}

Two main theoretical scenarios, based on accretion, are usually proposed to explain the formation of O-B type stars: 1) the core accretion model (McKee \& Tan 2002, 2003), where massive stars form from massive cores, and 2) the competitive accretion model (Bonnell et al. 2007), where a molecular cloud fragments into low-mass cores, which form stars that compete to accrete mass from a common gas reservoir. Both models predict the existence of accretion disks around massive young stellar objects (YSOs) and jets driving molecular outflows. The core accretion model is a scaled-up scenario of low-mass star formation: in this case, collimated jets and accretion disk should have the same properties as in low-mass YSOs. The competitive accretion model suggests that massive stars form in densely clustered environments and that disks and collimated jets are perturbed by interaction with stellar companions. To distinguish between these models, it would be helpful to obtain observation of disk/jet systems in a statistical sample of massive YSOs to assess the fraction of sources without such a system, and to derive their properties to compare with low-mass YSOs. Unlike the still-challenging direct observation of circumstellar disks, outflows/jets extend on larger scales and are easier to observe.

High-angular resolution studies of low-mass star-forming regions show that silicon monoxide $(\mathrm{SiO})$ thermal emission is the best tool for tracing jets: unlike other tracers such as CO, it suffers minimal contamination from swept-up cavities, and it allows one to probe obscured regions close to protostars (e.g. Codella et al. 2007). The formation of $\mathrm{SiO}$ can be attributed to the sputtering of Si atoms from charged grains in a magnetised C-shock with velocities $\geq 25 \mathrm{~km} \mathrm{~s}^{-1}$ (Schilke et al. 1997; Gusdorf et al. 2008a). Whether $\mathrm{SiO}$ emission originates from shocked ambient material or traces the primary jet itself is still a matter of debate (e.g., Cabrit et al. 2007, 2012, and references therein). While $\mathrm{SiO}$ is extensively observed in low-mass YSOs, high-angular resolution studies of $\mathrm{SiO}$ thermal emission in high-mass starforming regions are still limited to a handful of objects (Cesaroni et al. 1999; Hunter et al. 1999; Qiu et al. 2007; Zhang et al. 2007; Zapata et al. 2009) with a typical resolution of several thousand AU.

The prominent far-IR source IRAS $17233-3606$ is one of the best laboratories for studing massive star formation because of its close distance (1 kpc, Leurini et al. 2011a), high luminosity $\left(\sim 1.7 \times 10^{4} L_{\odot}\right)$, and relatively simple geometry. In a previous study in CO with the SMA (Leurini et al. 2009, hereafter Paper I), we resolved multiple outflows with high collimation factors, extremely high velocity (EHV) emission ( $\left|v-v_{\text {LSR }}\right|$ up to $200 \mathrm{~km} \mathrm{~s}^{-1}$ ), and flow parameters typical of massive YSOs. However, the severe contamination from swept-up components and the very elongated beam $\left(\sim 5^{\prime \prime} \times 3^{\prime \prime}\right)$ did not allow us to understand the number of outflows in the region or to identify the corresponding driving sources and to assess the occurrence of jets.

In this paper, we present $\mathrm{SMA} \mathrm{SiO}(5-4)$ and complementary APEX $\mathrm{SiO}(5-4)$ and (8-7) observations aimed at separating the outflow multiplicity in the cluster. We focus on the OF1 flow, which is associated with compact jet-like $\mathrm{H}_{2}$ emission at $2.12 \mu \mathrm{m}$ and EHV CO, and is possibly driven by a zero-age main-sequence (ZAMS) B1 star (Zapata et al. 2008). 


\section{Observations and data reduction}

\subsection{APEX}

We mapped a region of $70^{\prime \prime} \times 70^{\prime \prime}$ in the $\mathrm{SiO}(5-4)$ line at $217.105 \mathrm{GHz}$ and of $36^{\prime \prime} \times 36^{\prime \prime}$ in $\mathrm{SiO}(8-7)$ at $347.331 \mathrm{GHz}$ in raster mode with the APEX-1 and -2 receivers, respectively, on the $\mathrm{APEX}^{1}$ telescope in May 2010. The spacing between pointing centres was chosen to be $14^{\prime \prime}$ at $217 \mathrm{GHz}$ and 9" at $347 \mathrm{GHz}$. The centre of the maps was taken as $\alpha_{\text {[J2000] }}=17^{\mathrm{h}} 26^{\mathrm{m}} 42^{\mathrm{s}} .93$, $\beta_{[\mathrm{J} 2000]}=-36^{\circ} 09^{\prime} 20^{\prime \prime}$. The APEX facility fast Fourier transform spectrometers (Klein et al. 2006) allow a simultaneous observations of $1 \mathrm{GHz}$ bandwidth per receiver, centred on the $\mathrm{SiO}(5-4)$ and (8-7) lines. The velocity resolution of the original data is $\sim 0.3 \mathrm{~km} \mathrm{~s}^{-1}$ at $217 \mathrm{GHz}$ and $\sim 0.2 \mathrm{~km} \mathrm{~s}^{-1}$ at $347 \mathrm{GHz}$. Data were converted into $T_{\mathrm{MB}}$ units assuming a forward efficiency of 0.95 for both receivers, and a beam efficiency of 0.75 and 0.73 for the $\mathrm{SiO}(5-4)$ and (8-7) line, respectively. Pointing was checked on the $\mathrm{CO}(2-1)$ and (3-2) lines towards RAFGL4211, NGC 6072 and NGC 6302 and was found to have an rms of $\left(3{ }^{\prime \prime} 3,33^{\prime \prime} 8\right)$ for the APEX-1 data, and of (1". $\left.4,00^{\prime} 6\right)$ for the APEX-2 data. Data cubes were made with the XY_MAP task in the GILDAS software $^{2}$ with a common angular resolution of $300^{\prime} 5$. The final spectral resolution is $3.0 \mathrm{~km} \mathrm{~s}^{-1}$, with an rms of $0.02 \mathrm{~K}$ for $\mathrm{SiO}\left(8_{-7}\right)$ and $0.1 \mathrm{~K}$ for $\mathrm{SiO}(5-4)$.

\subsection{SMA}

SiO(5-4) line observations of IRAS 17233-3606 were obtained in June 2008 with the Submillimeter Array $^{3}$ (SMA) in its compact-north configuration with baselines ranging in projected length from 6 to $74 \mathrm{k} \lambda$. The central frequency of the lower sideband receivers was $217.1049 \mathrm{GHz}$, while the upper sideband central frequency was $227.1049 \mathrm{GHz}$. The full bandwidth of the SMA correlator was $4 \mathrm{GHz}$ (2 GHz in each band). The SMA digital correlator was configured to provide a spectral resolution of $0.406 \mathrm{MHz}\left(\sim 0.56 \mathrm{~km} \mathrm{~s}^{-1}\right)$. The primary beam at $217 \mathrm{GHz}$ has a FWHM diameter of $\sim 54^{\prime \prime}$.

The region was covered with a mosaic of two fields $\left(\alpha_{[\mathrm{J} 2000]}=17^{\mathrm{h}} 26^{\mathrm{m}} 42^{\mathrm{s}} .64, \delta_{[\mathrm{J} 2000]}=-36^{\circ} 09^{\prime} 18^{\prime \prime} .0, \alpha_{[\mathrm{J} 2000]}=\right.$ $\left.17^{\mathrm{h}} 26^{\mathrm{m}} 42 \mathrm{~s} .64, \delta_{[\mathrm{J} 2000]}=-36^{\circ} 09^{\prime} 38^{\prime \prime} \cdot 0\right)$. Bandpass calibration was made with $3 \mathrm{C} 279$. We used Titan for the flux calibration with an accuracy of $15-20 \%$, based on the SMA monitoring of quasars. The gain calibration was done via frequent observations of the quasar 1626-298. The zenith opacity, measured with the NRAO tipping radiometer located at the Caltech Submillimeter Observatory, was very stable at $\sim 0.2$, indicating reasonable weather conditions during the observations.

The initial flagging and calibration was performed with the IDL superset MIR ${ }^{4}$. The imaging and data analysis was conducted in MIRIAD (Sault et al. 1995) and GILDAS. Images were produced using natural weighting. The resulting synthesised beam is $3^{\prime \prime} .2 \times 2$.'5 with a PA of $16^{\circ}$. The final

\footnotetext{
1 APEX is a collaboration between the Max-Planck-Institut für Radioastronomie, the European Southern Observatory, and the Onsala Space Observatory.

2 http://wWw.iram.fr/IRAMFR/GILDAS

3 The Submillimeter Array (SMA) is a joint project between the Smithsonian Astrophysical Observatory and the Academia Sinica Institute of Astronomy and Astrophysics, and is funded by the Smithsonian Institution and the Academia Sinica.

4 The MIR cookbook by Charlie Qi can be found at

http: //cfa-www . harvard. edu/ cqi/mircook.html
}

rms corresponds to $0.1 \mathrm{Jy} /$ beam with a spectral resolution of $2 \mathrm{~km} \mathrm{~s}^{-1}$. The conversion factor between Jy/beam and $\mathrm{K}$ is 3.4.

\section{Observational results}

\subsection{SMA data}

The continuum emission of the region at $217 \mathrm{GHz}$ is shown in Fig. 1. We performed a 2-D Gaussian fit of the mm continuum emission, and determined its peak to be at $\alpha_{\mathrm{J} 2000}=$ $17^{\mathrm{h}} 26^{\mathrm{m}} 42^{\mathrm{s}} .506, \delta_{\mathrm{J} 2000}=-36^{\circ} 09^{\prime} 18^{\prime \prime} .319$ with positional errors on the fit of $\sim 0$. $^{\prime} 04$. The peak flux is $\sim 2.10 \pm 0.04 \mathrm{Jy} \mathrm{beam}^{-1}$, the integrated flux $6.69 \mathrm{Jy}$. The results agree very well with the continuum data at $230 \mathrm{GHz}$ presented by Leurini et al. (2011a).

Figure 2 shows the emission of the $\mathrm{SiO}(5-4)$ line observed with the SMA and integrated over three different velocity ranges: the ambient velocity and the blue- and red-shifted velocities, which include emission at low (LV) and high velocities (HV). The systemic velocity of the source is $-3.4 \mathrm{~km} \mathrm{~s}^{-1}$ (Bronfman et al. 1996). However large uncertainties in the determination of the ambient velocity were reported in previous SMA observations of complex molecules towards the peak of the millimetre continuum emission (Leurini et al. 2011b). Therefore, we conservatively assumed ambient emission (in green in Fig. 2) from -10 to $10 \mathrm{~km} \mathrm{~s}^{-1}$. Blue- and red-shifted emission is clearly detected towards at least two of the outflows identified in the region in Paper I, OF1 and OF2. SiO emission is also detected near the $\mathrm{H}_{2}$ emission to the south of the IRAS 17233-3606 cluster, thus supporting the scenario that these $\mathrm{H}_{2}$ structures are also associated with the outflows, as there is no $\mathrm{H}_{2}$ emission elsewhere in the map. Ambient emission is detected all along OF2 and towards the $\mathrm{H}_{2}$ bow to the north of IRAS 17233-3606. While in our previous study we suggested two possible axes for OF2 (Fig. 2, left panel), the $\mathrm{SiO}(5-4)$ data solve the degeneracy and clearly outline its axis (Fig. 2, right panel).

The blue- and red-shifted $\mathrm{SiO}$ emission shown in Fig. 2 concentrates along the axis of OF1 (see Fig. 1). The OF1 outflow shows interesting properties that deserve a closer look (Fig. 1). The position-velocity diagram of the $\mathrm{SiO}(5-4)$ line along the axis of OF1 is presented in Fig. 3: the $\mathrm{SiO}$ emission follows a linear velocity-distance relation, typically referred to as the Hubble-law, where the maximum radial velocity is proportional to position (e.g., Lada \& Fich 1996). The morphology of OF1 as traced by $\mathrm{SiO}$ is very similar to that pictured by $\mathrm{H}_{2}$. Its red lobe, observed in $\mathrm{H}_{2}$ and in EHV CO emission (up to $\sim 120 \mathrm{~km} \mathrm{~s}^{-1}$ ), is here confirmed by $\mathrm{SiO}$ red-shifted emission at $\mathrm{LV}$ and $\mathrm{HV}$ velocities (up to $\sim 55 \mathrm{~km} \mathrm{~s}^{-1}$ at a $3 \sigma$ level, see Fig. 3). Interestingly, the $\mathrm{HV} \mathrm{SiO} \mathrm{emission} \mathrm{peaks} \mathrm{between} \mathrm{the} \mathrm{apex} \mathrm{of} \mathrm{the} \mathrm{H}_{2}$ emission and the peak of the EHV CO emission. The blue lobe is traced by LV and $\mathrm{HV} \mathrm{CO}$ and $\mathrm{SiO}$ (see Fig. 1). The LV SiO emission is also very similar to that of $\mathrm{SO}\left(5_{6}-4_{5}\right)$ (see Fig. 1, left panel), which is the only tracer, together with $\mathrm{SiO}$, that clearly shows a bipolar morphology along OF1. Interestingly, the similarity between the $\mathrm{SiO}$ and $\mathrm{SO}$ emissions follows the recent result of Tafalla et al. (2010), who found that the $\mathrm{SiO}$ and SO molecules have very similar line profiles in collimated molecular outflows driven by Class 0 low-mass sources. Based on the FWHM of the LV blueand red-shifted $\mathrm{SiO}$ integrated emission, we infer an observed area of $\sim 30^{\prime \prime}$ squared and a length of $\sim 9$.' 6 , corresponding to a deconvolved area of $22^{\prime \prime}$ squared and to a deconvolved length of $\sim 9$ '. 0 . However, the emission is unresolved along the minor axis of OF1.

It is interesting to compare the $\mathrm{SiO}$ and $\mathrm{H}_{2}$ images of the OF1 outflow with the recent findings towards typical low-mass 


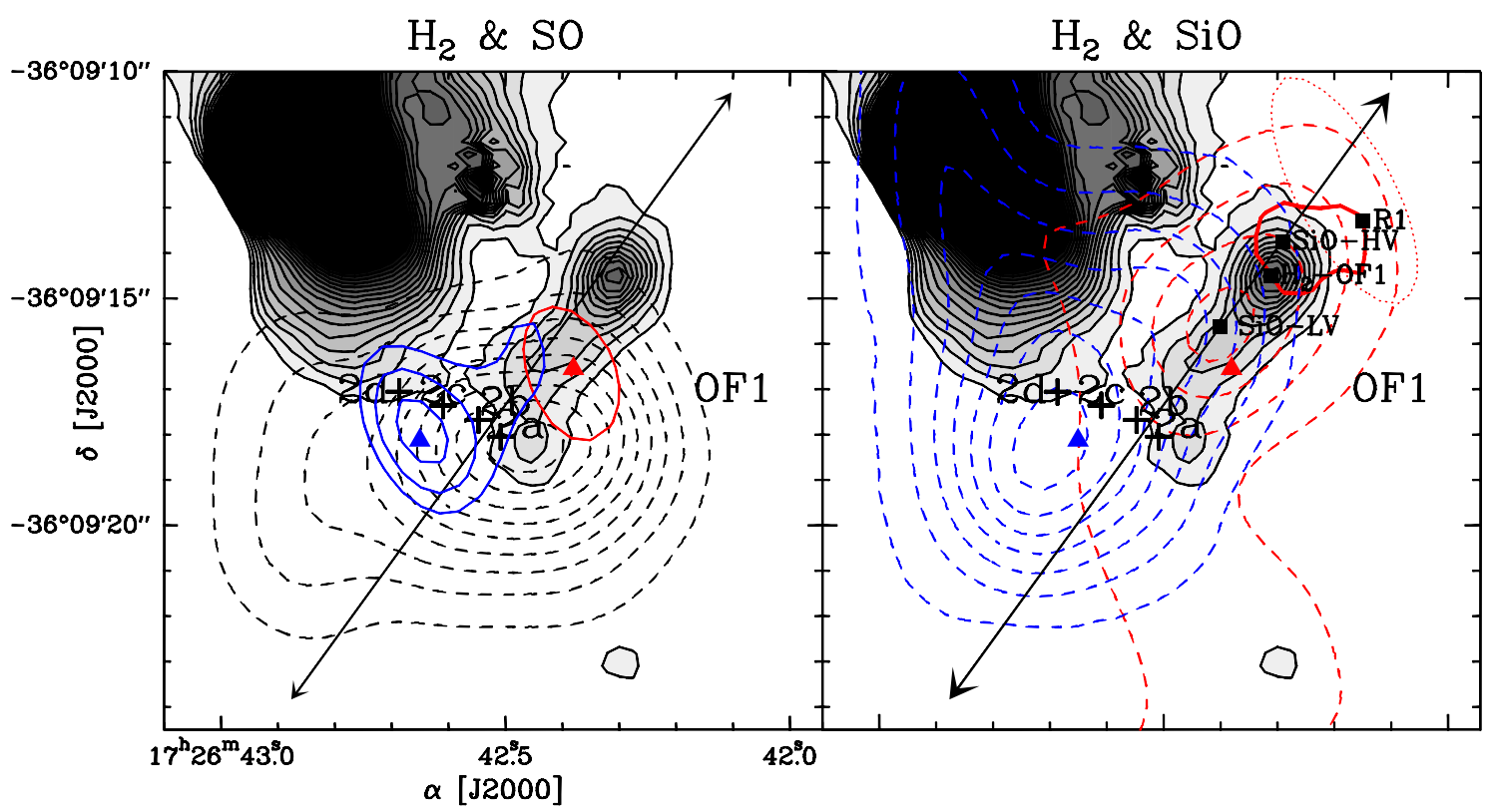

Fig. 1. Left: dashed contours (from $10 \sigma, 0.23 \mathrm{Jy} /$ beam, in step of $10 \sigma$ ) are the $1.4 \mathrm{~mm}$ continuum emission observed with the SMA. The solid blue and red contours are the $\mathrm{SO}\left(5_{6}-4_{5}\right)$ blue- and red-shifted emission from Paper I (blue: from 80 to $104 \mathrm{Jy}_{\text {beam }}^{-1}$ in steps of $10 \mathrm{Jy}$ beam ${ }^{-1}$; red: 10 and $12 \mathrm{Jy} \mathrm{beam}^{-1}$ ). Right: integrated emission of the $\mathrm{SiO}(5-4)$ line: the dashed blue and red contours are the LV blue- and red-shifted SiO emission $\left(v_{\mathrm{bl}}=[-20,-10] \mathrm{km} \mathrm{s}^{-1}\right.$, from $5 \sigma(2.5 \mathrm{Jy} / \mathrm{beam})$ in step of $10 \sigma ; v_{\mathrm{rd}}=[+10,+39] \mathrm{km} \mathrm{s}^{-1}$, from $5 \sigma(4 \mathrm{Jy} /$ beam $)$ in step of $\left.10 \sigma\right)$, the thick contour the HV red-shifted emission $\left(v_{\mathrm{rd}}=[+50,+70] \mathrm{km} \mathrm{s}^{-1}, 4 \sigma(2.4 \mathrm{Jy} / \mathrm{beam})\right)$. The red dotted contour is the EHV CO $(2-1)$ red-shifted emission (see Fig. 3 of Paper I). The black squares mark the peak positions of the EHV CO(2-1) (R1) red-shifted emission, of the SiO(5-4) LV ( $\mathrm{SiO}-\mathrm{LV}$ ) and $\mathrm{HV}$ red-shifted emission (SiO-HV), and of the $\mathrm{H}_{2}$ emission in OF1 $\left(\mathrm{H}_{2}-\mathrm{OF} 1\right)$. In both panels, the grey scale and the solid black contours represent the $\mathrm{H}_{2}$ emission at $2.12 \mu \mathrm{m}$. The black crosses mark the positions of the VLA-2a,-2b,-2c and $-2 \mathrm{~d} 1.3 \mathrm{~cm}$ continuum sources (Zapata et al. 2008); the arrow marks the OF1 outflow. The blue and red triangles mark the peaks of the blue- and red-shifted $\mathrm{SO}\left(5_{6}-4_{5}\right)$ emission.

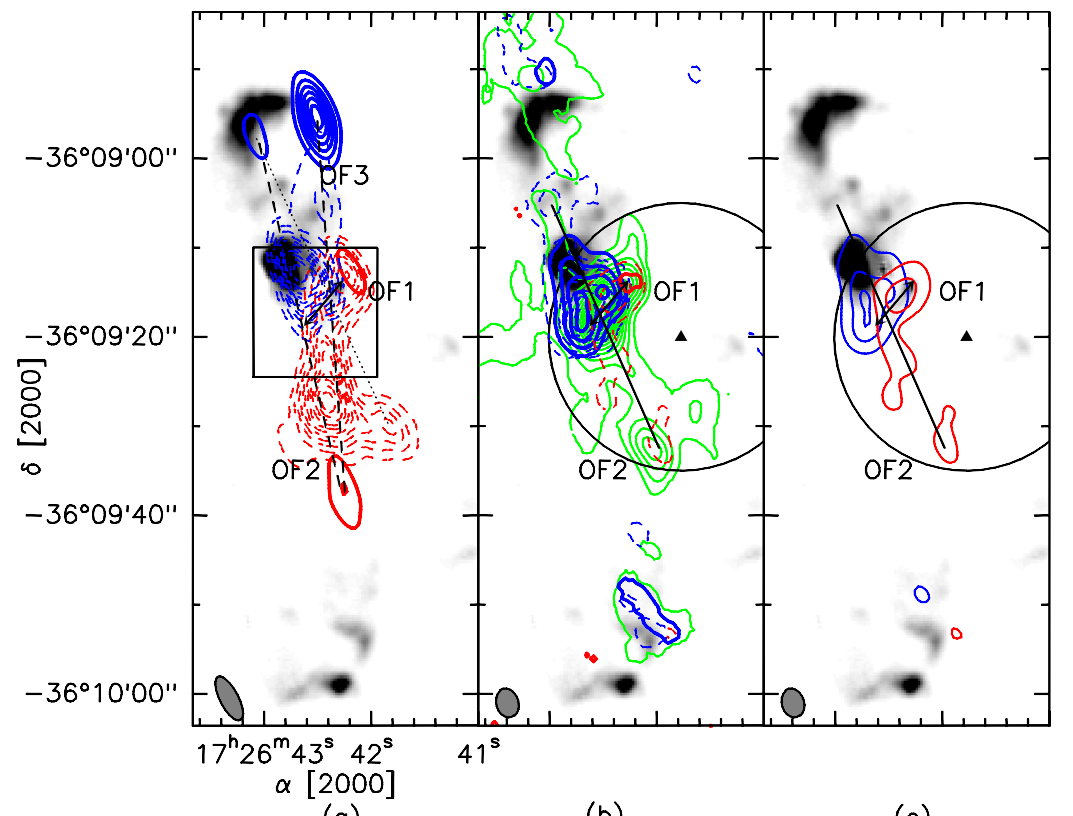

(a)

(b)

(c)

Fig. 2. Left panel: integrated emission of the $\mathrm{CO}(2-1)$ blue- and red-shifted wings. The solid contours show the EHV blue- and red-shifted emission; the dashed contours mark the HV blue- and red-shifted emission (see Paper I, Fig. 3). The dashed and solid lines outline the possible molecular outflows (OF1, OF2, OF3); the dotted line marks the alternative direction of the OF2 outflow. The central box is the region mapped in Fig. 1. Middle panel: Integrated emission of the $\mathrm{SiO}(5-4)$ line: the dashed blue and red contours are the LV blue- and red-shifted emission $\left(v_{\mathrm{bl}}=[-20,-10] \mathrm{km} \mathrm{s}^{-1}\right.$, from $5 \sigma(2.5 \mathrm{Jy} /$ beam $)$ in steps of $10 \sigma ; v_{\mathrm{rd}}=[+10,+39] \mathrm{km} \mathrm{s}^{-1}$, from $5 \sigma(6 \mathrm{Jy} / \mathrm{beam})$ in steps of $\left.10 \sigma\right)$, the thick contours the HV blue- and red-shifted emission $\left(v_{\mathrm{bl}}=[-50,-20] \mathrm{km} \mathrm{s}^{-1}\right.$, from $5 \sigma(4 \mathrm{Jy} / \mathrm{beam})$ in steps of $\left.10 \sigma ; v_{\mathrm{rd}}=[+50,+70] \mathrm{km} \mathrm{s}{ }^{-1}, 4 \sigma(2.4 \mathrm{Jy} / \mathrm{beam})\right)$. The green contours are the integrated emission at ambient velocities $\left(v_{\mathrm{gr}}=[-10,+10] \mathrm{km} \mathrm{s}^{-1}\right.$; from $5 \sigma(3 \mathrm{Jy} / \mathrm{beam})$ in steps of $\left.10 \sigma\right)$. Right panel: integrated emission of the $\mathrm{SiO}(5-4)$ line in the velocity ranges used for modelling the $\mathrm{SiO}$ emission in Sect. 4: the blue and red contours are the blue- and red-shifted emission in the ranges $\left(v_{\mathrm{bl}}=[-30,-20] \mathrm{km} \mathrm{s}^{-1}\right.$, from $5 \sigma(4 \mathrm{Jy} / \mathrm{beam})$ in steps of $10 \sigma ; v_{\mathrm{rd}}=[+10,+39] \mathrm{km} \mathrm{s}{ }^{-1}$, from $5 \sigma(6.5 \mathrm{Jy} /$ beam $)$ in steps of $10 \sigma)$. In the three panels, the solid line marks the axis of OF2 as identified in SiO. The arrowed line marks that of $\mathrm{OF} 1$. The solid circle represents the APEX $30^{\prime}, 5$ beam centred on $\alpha_{[2000]}=17^{\mathrm{h}} 26^{\mathrm{m}} 41^{\mathrm{s}} .77, \delta_{[2000]}=-36^{\circ} 09^{\prime} 20^{\prime \prime}$, the position used in Sect. 4 for our modelling of the $\mathrm{SiO}$ emission ( $\mathrm{SiO}-\mathrm{APEX}$, see Table 2) and here labelled by a black triangle. In both panels, the grey scale represents the $\mathrm{H}_{2}$ emission at $2.12 \mu \mathrm{m}$ (Paper I). 


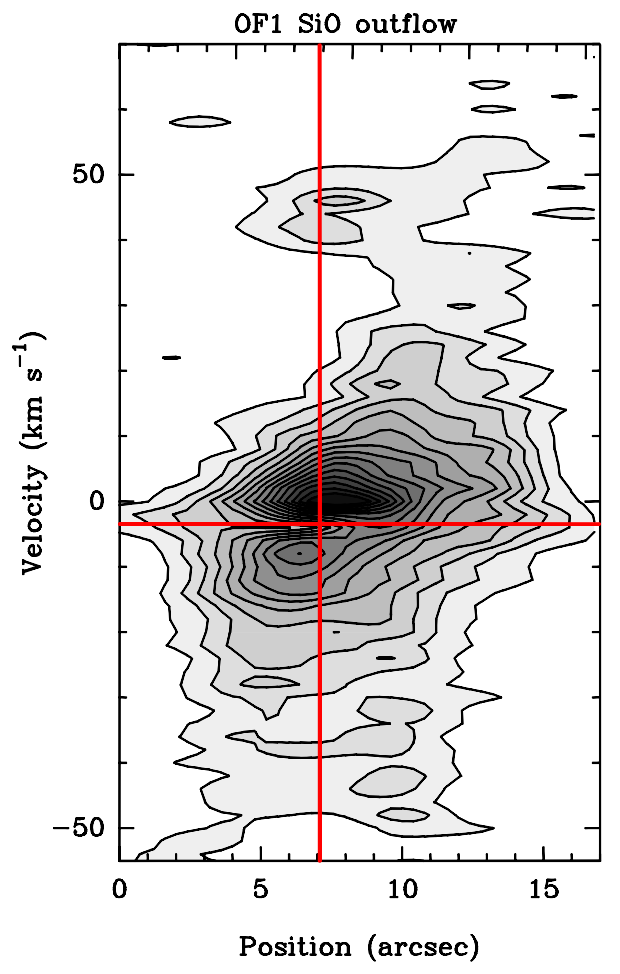

Fig. 3. Position-velocity diagram of the $\mathrm{SiO}(5-4)$ transition (SMA data) along the OF1 axis shown in Fig. 1. The vertical line marks the $\mathrm{mm}$ continuum peak, the horizontal line the ambient velocity $\left(-3.4 \mathrm{~km} \mathrm{~s}^{-1}\right.$, Bronfman et al. 1996). Contours are from $2 \sigma$ (0.2 Jy/beam) in steps of $4 \sigma$.

protostars such as HH211 and HH212 (see e.g., Hirano et al. 2006; Codella et al. 2007) located at 300-500 pc distance. The $\mathrm{SiO}$ emission in HH211 and HH212 is confined to a highly collimated bipolar jet, well detected thanks to sub-arcsec spatial resolution of the SMA and Plateau de Bure images. The SiO jet can be traced to within 500-1000 AU of the protostar, in a region that is heavily obscured in $\mathrm{H}_{2}$ images. On the other hand, the brightest $\mathrm{H}_{2}$ knots are located in an outer region and lack a well-defined $\mathrm{SiO}$ counterpart, probably tracing more powerful shock where $\mathrm{SiO}$ does not form/survive or where the $\mathrm{SiO}$ excitation is extremely high and consequently the low-J mmtransitions are weak. In addition, the position-velocity diagrams suggest that the $\mathrm{SiO}$ emission increases in velocity with increasing distance from the driving source. The present OF1 images, although observed with a poorer spatial resolution, seem to suggest a similar picture: (i) the position of the $\mathrm{SiO}$ red lobe is not correlated with the brightest $\mathrm{H}_{2}$ knot, and (ii) the SiO-LV emission peaks closer to the driving source than the SiO-HV peak. Only future higher spatial resolution observations, possibly with ALMA, will allow a proper characterisation of the region.

Comparisons between the SMA SiO(5-4) flux and the flux observed with the APEX telescope in the same transition are discussed in the next section.

\subsection{APEX data}

Figure 4 shows the $\mathrm{SiO}(5-4)$ and (8-7) maps from APEX at their original angular resolution, 30.'5 for $\mathrm{SiO}(5-4)$ and 19.'1 for $\mathrm{SiO}(8-7)$. Given the low resolution of the APEX data, the emission from the different outflows in the region is not resolved. When averaged to the common resolution of 30.'5, both $\mathrm{SiO}(8-7)$ and (5-4) maps show a maximum at $\alpha_{[\mathrm{J} 2000]}=$ $17^{\mathrm{h}} 26^{\mathrm{m}} 41^{\mathrm{s}} .77, \delta_{[\mathrm{J} 2000]}=-36^{\circ} 09^{\prime} 20^{\prime \prime}$, the centre of the circle
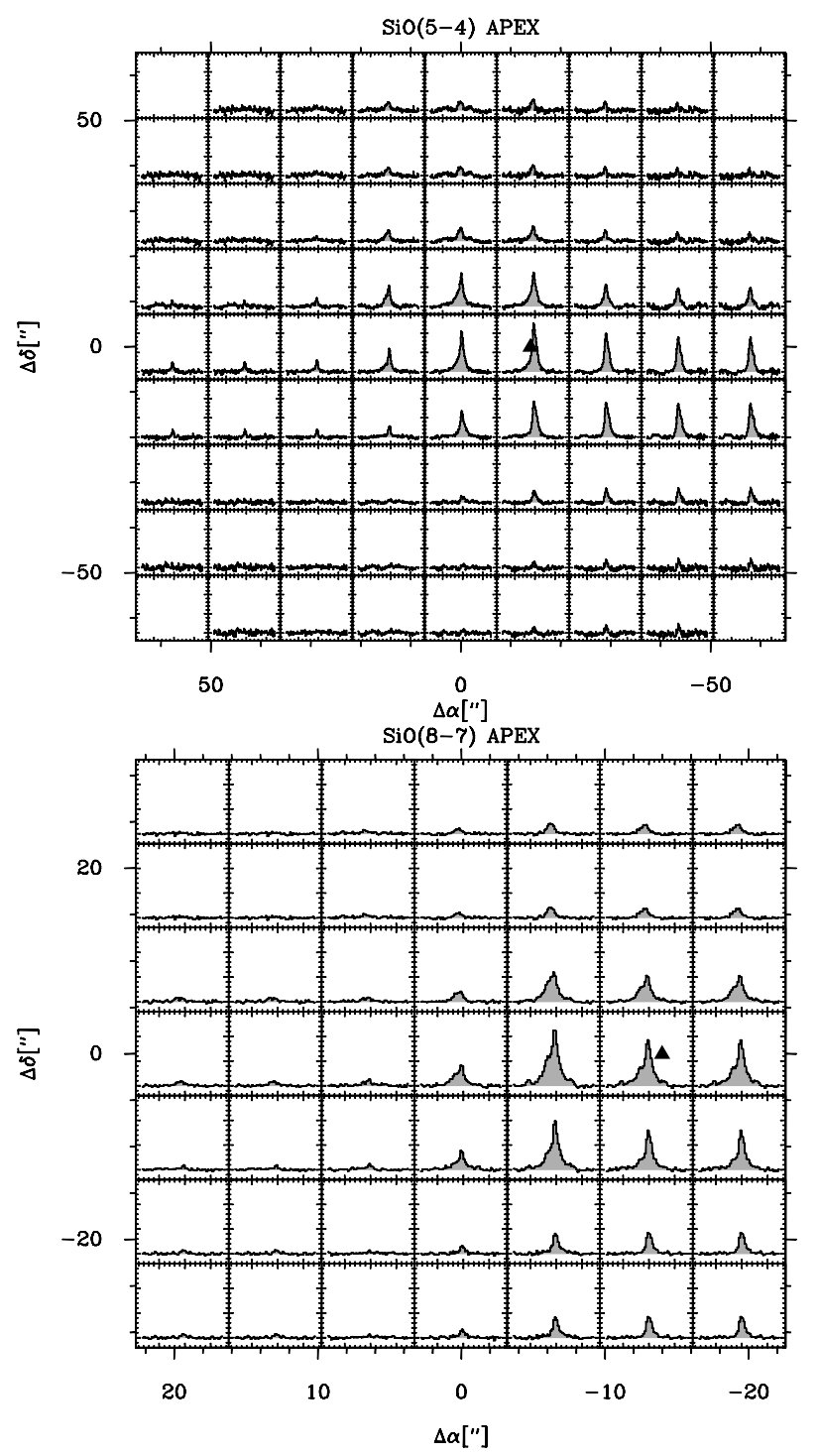

Fig. 4. $\mathrm{SiO}(5-4)$ (top panel) and (8-7) (bottom panel) maps observed with the APEX telescope. The velocity axis extendes from -95 to $85 \mathrm{~km} \mathrm{~s}^{-1}$ for both data sets, while the temperature scale is covers -0.4 to $3 \mathrm{~K}$. The data are shown with the original angular resolution of $30.5(\mathrm{SiO}(5-4))$ and $19.1(\mathrm{SiO}(8-7))$. The centre of the maps is given in Table 1. The black triangle marks the peak position (SiO-APEX, see Table 2) of the integrated intensity $\mathrm{SiO}(5-4)$ and (8-7) maps (in the velocity range $-40<v<40 \mathrm{~km} \mathrm{~s}^{-1}$ ) smoothed to the angular resolution of the $\mathrm{SiO}(5-4)$ data, $300^{\prime} 5$.

(representing the APEX beam) partially seen in Fig. 2 (right panel). This position has no counterpart in other tracers: it is situated at $\sim\left(-9^{\prime \prime},-2^{\prime \prime}\right)$ from VLA-2a, the strongest $\mathrm{cm}$ source of the region, and at $\sim\left(-8^{\prime \prime},-4^{\prime \prime}\right)$ from the centre of the $\mathrm{LV} \mathrm{SiO}$ red-shifted emission in OF1. We believe, however, that the offset between SiO SMA and APEX data is due to the poor sampling of the APEX map. In Fig. 5 we present the $\operatorname{SiO}(5-4)$ emission observed with APEX in three different velocity ranges (total emission, red-shifted and blue-shifted emission). The map clearly shows that $\mathrm{SiO}$ is spread over a large area and is associated with several shocks from the IRAS 17233-3606 cluster detected in $\mathrm{H}_{2}$.

The APEX spectra extracted at the peak of the single-dish $\mathrm{SiO}$ emission are shown in Fig. 6. Although the APEX beam at this position covers emission close to the ambient velocity from OF1 and OF2, the red- and the blue-shifted emission is 
Table 1. Summary of the observations.

\begin{tabular}{|c|c|}
\hline \multicolumn{2}{|r|}{ SMA } \\
\hline Centre of the map & $\begin{array}{cc}\alpha_{\text {J2000 }} & 17^{\mathrm{h}} 26^{\mathrm{m}} 42^{\mathrm{s}} .651 \\
\delta & -36^{\circ} 09^{\prime} 38^{\prime \prime} 00\end{array}$ \\
\hline Beam size & $3{ }^{\prime \prime} 2 \times 2,5$, PA $16^{\circ}$ \\
\hline Central frequency & $217104.980 \mathrm{MHz}$ \\
\hline Velocity resolution & $2 \mathrm{~km} \mathrm{~s}^{-1}$ \\
\hline Bandwidth & $2 \mathrm{GHz}$ \\
\hline \multicolumn{2}{|r|}{ APEX } \\
\hline Centre of the map & $\begin{array}{c}\alpha_{\mathrm{J} 2000} 17^{\mathrm{h}} 26^{\mathrm{m}} 42^{\mathrm{s}} .930 \\
\delta_{\mathrm{J} 2000}-36^{\circ} 09^{\prime} 20^{\prime} 00\end{array}$ \\
\hline Beam size & 30.5 \\
\hline Central frequency ${ }^{a}$ & $217104.9800 \mathrm{MHz}, 347330.5786 \mathrm{MHz}$ \\
\hline Velocity resolution $^{a}$ & $0.3 \mathrm{~km} \mathrm{~s}^{-1}, 0.2 \mathrm{~km} \mathrm{~s}^{-1}$ \\
\hline Bandwidth & $1 \mathrm{GHz}$ \\
\hline
\end{tabular}

Notes. ${ }^{(a)}$ for $\mathrm{SiO}(5-4)$ and (8-7), respectively.

Table 2. Summary of relevant positions in the IRAS 17233-3606 cluster.

\begin{tabular}{|c|c|c|}
\hline mm-continuum & $\alpha_{[\mathrm{J} 2000]}=17^{\mathrm{h}} 26^{\mathrm{m}} 42^{\mathrm{s}} .51$ & $\delta_{[\mathrm{J} 2000]}=-36^{\circ} 09^{\prime} 18^{\prime \prime} .32$ \\
\hline VLA-2a & $\alpha_{[\mathrm{J} 2000]}=17^{\mathrm{h}} 26^{\mathrm{m}} 42^{\mathrm{s}} .51$ & $\delta_{[\mathrm{J} 2000]}=-36^{\circ} 09^{\prime} 18^{\prime \prime} .^{\prime} 04$ \\
\hline VLA-2b & $\alpha_{\text {[J2000] }}=17^{\mathrm{h}} 26^{\mathrm{m}} 42^{\mathrm{s}} .55$ & $\delta_{[\mathrm{J} 2000]}=-36^{\circ} 09^{\prime} 17^{\prime \prime} .67$ \\
\hline VLA-2c & $\alpha_{[\mathrm{J} 2000]}=17^{\mathrm{h}} 26^{\mathrm{m}} 42^{\mathrm{s}} .61$ & $\delta_{[\mathrm{J} 2000]}=-36^{\circ} 09^{\prime} 17^{\prime \prime} .35$ \\
\hline VLA-2d & $\alpha_{[\mathrm{J} 2000]}=17^{\mathrm{h}} 26^{\mathrm{m}} 42^{\mathrm{s}} .69$ & $\delta_{[\mathrm{J} 2000]}=-36^{\circ} 09^{\prime} 17^{\prime \prime} .05$ \\
\hline SiO-APEX & $\alpha_{[\mathrm{J} 2000]}=17^{\mathrm{h}} 26^{\mathrm{m}} 41^{\mathrm{s}} .77$ & $\delta_{[\mathrm{J} 2000]}=-36^{\circ} 09^{\prime} 20^{\prime} 00$ \\
\hline $\mathrm{H}_{2}-\mathrm{OF} 1$ & $\alpha_{[\mathrm{J} 2000]}=17^{\mathrm{h}} 26^{\mathrm{m}} 42^{\mathrm{s}} \cdot 31$ & $\delta_{[\mathrm{J} 2000]}=-36^{\circ} 09^{\prime} 14^{\prime \prime} \cdot 49$ \\
\hline $\mathrm{SiO}-\mathrm{HV}^{\mathrm{a}}$ & $\alpha_{[\mathrm{J} 2000]}=17^{\mathrm{h}} 26^{\mathrm{m}} 42^{\mathrm{s}} .29$ & $\delta_{[\mathrm{J} 2000]}=-36^{\circ} 09^{\prime} 13^{\prime \prime} .74$ \\
\hline $\mathrm{SiO}-\mathrm{LV}^{\mathrm{b}}$ & $\alpha_{[\mathrm{J} 2000]}=17^{\mathrm{h}} 26^{\mathrm{m}} 42^{\mathrm{s}} .40$ & $\delta_{[\mathrm{J} 2000]}=-36^{\circ} 09^{\prime} 15^{\prime \prime} .62$ \\
\hline $\mathrm{R} 1^{\mathrm{c}}$ & $\alpha_{[\mathrm{J} 2000]}=17^{\mathrm{h}} 26^{\mathrm{m}} 42^{\mathrm{s}} .15$ & $\delta_{[\mathrm{J} 2000]}=-36^{\circ} 09^{\prime} 13^{\prime \prime} \cdot 28$ \\
\hline
\end{tabular}

Notes. ${ }^{(a)}$ peak of the HV red-shifted SMA-SiO emission (Fig. 1); (b) peak of the LV red-shifted SMA-SiO emission (Fig. 1); ${ }^{(c)}$ peak of the EHV red-shifted CO emission (see Paper I and Fig. 1).

dominated by OF1 both spatially and in intensity, as discussed in Sect. 3.3. Therefore, in the following we assume that the spectra shown in Fig. 6 are associated with OF1 only and represent its integrated spectra over the 30.' 5 APEX beam.

The $\mathrm{SiO}(8-7)$ line shows bright wings extending to $\pm 40 \mathrm{~km} \mathrm{~s}^{-1}$, i.e. velocities slightly higher than those of the $\mathrm{SiO}(5-4)$ transition. Fig. 6 also shows that the $\mathrm{SiO}(8-7) /$ $\mathrm{SiO}(5-4)$ intensity ratio remains almost constant $(\sim 0.8)$ at ambient velocities while it increases to values around 1.7 at redshifted velocities, and 2.2 in the blue wing. If we assume that both $\mathrm{SiO}$ lines are tracing the same gas, this result suggests an increase of excitation conditions (discussed in Sect. 4) at high velocities, similar to the results obtained towards the two lowmass Class 0 sources L1448-mm and L1157-mm (Nisini et al. 2007) through $\mathrm{SiO}$ observations.

\subsection{Comparison between the SMA and the APEX $\mathrm{SiO}$ emission}

We compared the $\mathrm{SiO}(5-4)$ APEX spectrum at the centre of the single-dish map with the corresponding SMA spectrum integrated over the APEX beam. The SMA total flux corresponds to $41 \%$ of the single-dish emission, $57 \%$ in the blue-wings (which dominate the spectrum at this position), and $\sim 34 \%$ at ambient velocities. Given the broad band of the APEX and of the SMA receivers and the richness of the molecular spectrum of IRAS 17233-3606 (Leurini et al. 2008, and Paper I), several spectral features are detected simultaneously to $\mathrm{SiO}(5-4)$ in

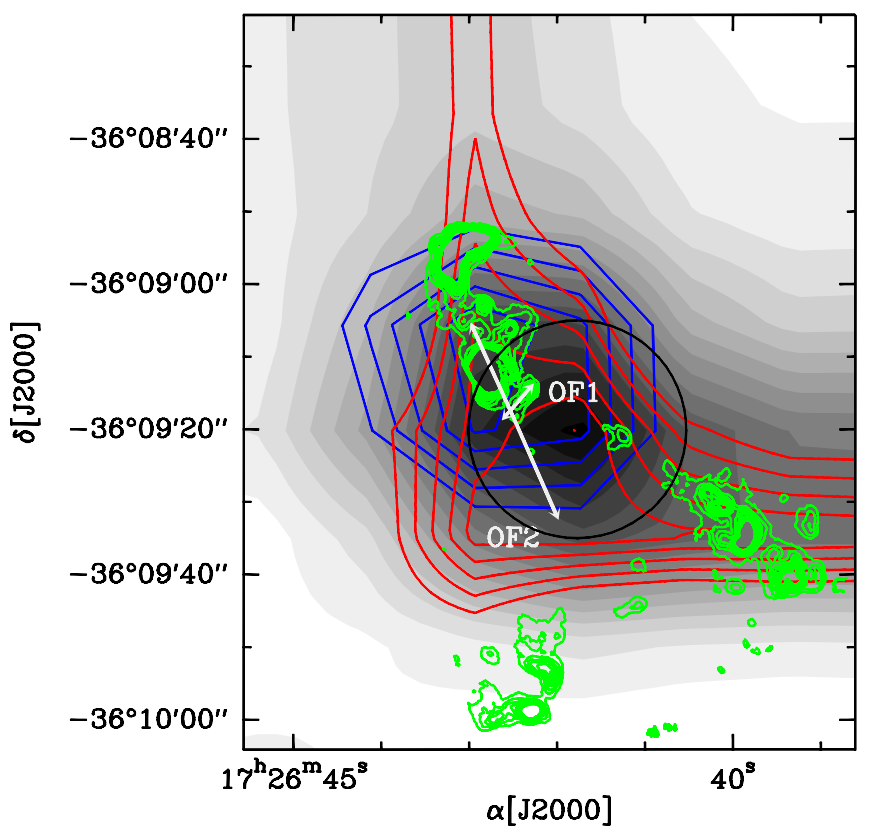

Fig. 5. Total integrated intensity $\left(-40<v<40 \mathrm{~km} \mathrm{~s}^{-1}\right.$; grey shades are from $10 \%$ of the peak emission, $43 \mathrm{~K} \mathrm{k} \mathrm{ms}^{-1}$, in steps of $5 \%$ ) of the $\mathrm{SiO}(5-4)$ transition observed with APEX. The red and blue contours are the red- $\left(5<v<40 \mathrm{~km} \mathrm{~s}^{-1}\right)$ and blue-shifted $\left(-40<v<-5 \mathrm{~km} \mathrm{~s}^{-1}\right)$ integrated emission observed with APEX (contours are 50\% of the peak emission $\left(9.4 \mathrm{~K} \mathrm{k} \mathrm{ms}^{-1}\right.$ for the red emission, $17.6 \mathrm{~K} \mathrm{k} \mathrm{ms}^{-1}$ for the blue emission) in steps of $10 \%$ ). The green contours represent the $2.12 \mu \mathrm{m}$ $\mathrm{H}_{2}$ emission. The white arrows label the OF1 and OF2 outflows. The black circle marks the APEX beam centred on the peak of the APEX $\mathrm{SiO}$ emission, as in Fig. 2.

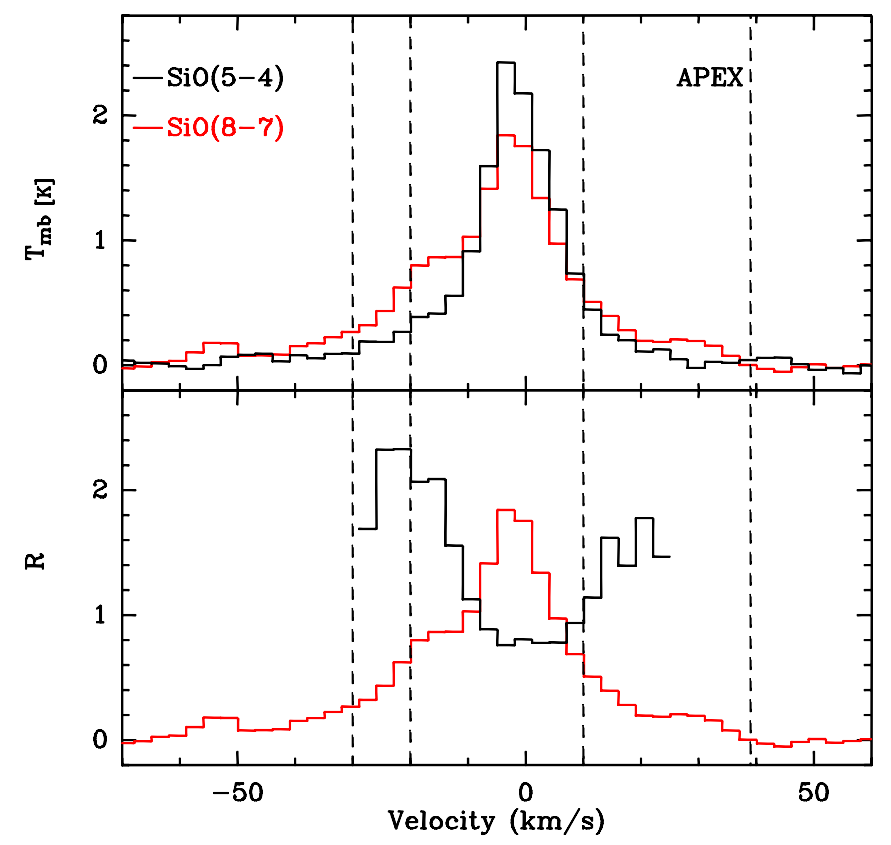

Fig. 6. Upper panel: APEX spectra of the $\mathrm{SiO}(5-4)$ and (8-7) lines in the OF1 region around IRAS 17233-3606 observed at $\alpha_{[\mathrm{J} 2000]}=$ $17^{\mathrm{h}} 26^{\mathrm{m}} 41^{\mathrm{s}} .77, \delta_{[\mathrm{J} 2000]}=-36^{\circ} 09^{\prime} 20^{\prime \prime}$. Lower panel: $\mathrm{SiO}(8-7) / \mathrm{SiO}(5-4)$ line ratio as function of velocity (black line). The red line is the $\mathrm{SiO}(8-7)$ spectrum. The dashed lines mark the velocity ranges used in Sect. 4 , blue $[-30,-20]$, and red $[+10,+39] \mathrm{km} \mathrm{s}^{-1}$.

both datasets. For emission in the band from complex molecules, most likely probing the hot core, which is expected to be compact, fluxes between 80 and $100 \%$ are recovered, hence 


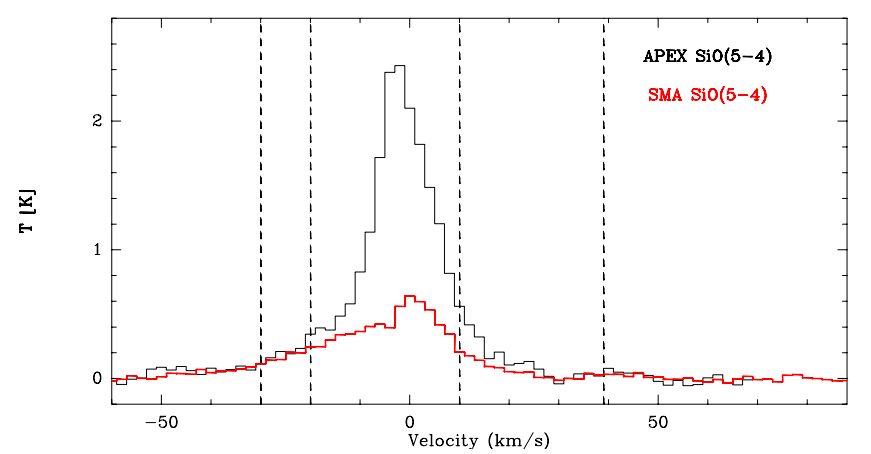

Fig. 7. $\mathrm{SiO}(5-4)$ spectra from APEX (black line) and the SMA (red line) observed towards OF1 at $\alpha_{[\mathrm{J} 2000]}=17^{\mathrm{h}} 26^{\mathrm{m}} 41.77, \delta_{[\mathrm{J} 2000]}=$ $-36^{\circ} 09^{\prime} 20^{\prime \prime}$. The dashed lines mark the velocity ranges used in Sect. 4 for modelling the $\mathrm{SiO}$ emission, blue $[-30,-20]$, and red $[+10,+39] \mathrm{km} \mathrm{s}^{-1}$.

confirming that the difference between the SMA and APEX SiO spectra is due to missing flux and not to calibration issues.

We also compared the SMA spectrum integrated over the APEX beam and centred on the peak of the APEX SiO emission with the corresponding APEX spectrum. The overlay of the two spectra is shown in Fig. 7. The SMA recovers $98 \%$ and $68 \%$ of the APEX flux in the red $\left([+10,+39] \mathrm{km} \mathrm{s}^{-1}\right)$ and blue low-velocity $\left([-20,-10] \mathrm{km} \mathrm{s}^{-1}\right)$ ranges plotted in Fig. 2b. In the APEX spectrum, emission at high-velocity range $\left(v_{\mathrm{rd}}=[+50,+70] \mathrm{km} \mathrm{s}^{-1}\right.$ and $\left.v_{\mathrm{bl}}<-30 \mathrm{~km} \mathrm{~s}^{-1}\right)$ is not detected. However, emission is detected at high velocities in the $[-30,-20] \mathrm{km} \mathrm{s}^{-1}$ range. In this range of velocities, the SMA recovers the whole APEX flux (Fig. 7). Therefore, in the range of velocities $[+10,+39] \mathrm{km} \mathrm{s}^{-1}$ and $[-30,-20] \mathrm{km} \mathrm{s}^{-1}$, the SMA data are not affected by filtering of large structures and the morphology of Fig. 2c (which shows the SMA SiO integrated emission in the ranges $[+10,+39] \mathrm{km} \mathrm{s}^{-1}$ and $\left.[-30,-20] \mathrm{km} \mathrm{s}^{-1}\right)$ is reliable. We show in Appendix A a joint deconvolution of the inteferometer and single-dish data performed on integrated intensity maps. However, combining the inteferometer data with the single-dish map is not needed for our purposes because the modelling of the $\mathrm{SiO}$ emission (see Sect. 4) is limited to the velocity ranges $[+10,+39] \mathrm{km} \mathrm{s}^{-1}$ and $[-30,-20] \mathrm{km} \mathrm{s}^{-1}$ where the SMA recovers the whole flux observed with APEX.

To estimate the contribution of OF1 to the APEX spectrum at the peak of the $\mathrm{SiO}$ emission, we computed the SMA fluxes integrated over the APEX beam centred on $\alpha_{[\mathrm{J} 2000]}=17^{\mathrm{h}} 26^{\mathrm{m}} 41^{\mathrm{s}} .77$, $\delta_{\text {[J2000] }}=-36^{\circ} 09^{\prime} 20^{\prime \prime}$ and over a region enclosing OF1. The blue-shifted $\left([-30,-20] \mathrm{km} \mathrm{s}^{-1}\right)$ SMA flux associated with OF1 is $66 \mathrm{Jy}$, corresponding to $66 \%$ of the total blue-shifted flux over the APEX beam. Similarly, the red-shifted $\left([+10,+39] \mathrm{km} \mathrm{s}^{-1}\right)$ SMA flux in OF1 is $71 \mathrm{Jy}$ ( $53 \%$ of the total flux over the APEX beam). Therefore, OF1 contributes up to $\sim 60 \%$ to the integrated $\mathrm{SiO}$ emission observed with APEX in the velocity ranges used in Sect. 4 for the modelling of the $\mathrm{SiO}$ spectra.

\section{SiO modelling}

In principle, specific modelling complications arise in the context of high-mass star formation, where the strong radiation field of the central object may affect both the physical and chemical structure of the shock and the excitation of molecules in the outflow region (such effects on the shock models are currently being studied, but their influence has not been properly assessed yet). The potential multiplicity of the sources or their outflows is also likely to generate modelling difficulties. Owing to the lack of information on the region, and as a first step towards a more complete description, we decided to model our $\mathrm{SiO}$ observations in two ways that are classically used to describe the $\mathrm{SiO}$ emission arising from shock regions generated in outflows of low-mass forming stars (e.g., Nisini et al. 2007; Gusdorf et al. 2011). The first one, hereafter referred to as "shock-LVG", is described in Sect. 4.1 and is based on the combination of a shock code and a radiative transfer code based on the large-velocitygradient (LVG) approximation. The second one (Sect. 4.2), hereafter referred to as "slab-LVG", consists of a simpler LVG description. In the first one, the shock is approximated by a layer of points with varying physical and chemical conditions from one point to another. In the second, the usual LVG physical parameters are given for one point that epitomises the whole layer of the shock-LVG method. Although still distant from the probably complex "real" configuration of multiple shocks at work within the APEX beam, we believe that this two-sided approach, especially the shock-modelling one, is the most rigorous so far available to constrain the abundance of $\mathrm{SiO}$ and link it to the mass of the associated outflow. Indeed, from a computational point of view, it is still impossible to design a model that would account for the precise geometrical, dynamical, physical, and chemical structure of the region. Moreover, no observational facility can currently provide the very high-angular resolution necessary to separate, for instance, a "wind shock" from an "ambient shock" component identified in the idealistic models presented, e.g., by Hollenbach (1997).

\subsection{Shock-LVG analysis}

We first base our modelling of the $\mathrm{SiO}$ emission on the C-type shocks interpretation presented in details in Gusdorf et al. (2008a). The $\mathrm{SiO}$ emission is accounted for by the combination of two codes: a state-of-the-art shock model and a radiativetransfer code based on the LVG approximation. The two codes codes are presented in detail in Gusdorf et al. (2008a,b, 2011). We nevertheless describe the main elements relevant to our study in the following paragraph.

The shock code calculates the dynamical, physical, and chemical structure of one-dimensional, stationary C-type shock models. The fractional abundances of over 125 species, linked by over 1000 reactions, are computed at each point of the shock. The gas-phase abundance of $\mathrm{H}_{2}$ is calculated, based mostly on the treatment described in Le Bourlot et al. (2002). The local abundance of $\mathrm{H}_{2}$ is of particular importance in the context of $\mathrm{SiO}$ modelling, as it is the most abundant species to collisionally excite $\mathrm{SiO}$. The excitation of its own 150 first levels (the most likely to be populated in the considered shocks) is also provided, making use of the $\mathrm{H}-\mathrm{H}_{2}$ collisional rate coefficients from Wrathmall et al. (2007). Specific grain processes, listed in Flower \& Pineau des Forêts (2003), are taken into account. In particular, in C-shock models, the grains undergo the sputtering impact of neutral particles at the speed of the ambipolar diffusion. Because the grain cores are partly made from silicon-bearing material, this sputtering leads to the release of elemental Si in the gas phase (Caselli et al. 1997; Schilke et al. 1997; May et al. 2000), where it reacts with $\mathrm{O}_{2}$ and $\mathrm{OH}$ to form $\mathrm{SiO}$. In the shock code, the rates of these two reactions are identical and correspond to the low-temperature measurements of the $\mathrm{Si}+\mathrm{O}_{2} \longrightarrow \mathrm{SiO}+\mathrm{O}$ reaction by Le Picard et al. (2001). Less prominent routes to the formation of $\mathrm{SiO}$, such as $\mathrm{Si}^{+}(\mathrm{OH}, \mathrm{H}) \mathrm{SiO}^{+}\left(\mathrm{H}_{2}, \mathrm{H}\right) \mathrm{HSiO}^{+}(\mathrm{e}, \mathrm{H}) \mathrm{SiO}$, are also included in our shock code. 
The magnetic field parameter, $b$, defined by $B(\mu \mathrm{G})=b \times$ $\sqrt{n_{\mathrm{H}}\left(\mathrm{cm}^{-3}\right)}$ where $n_{\mathrm{H}}$ is the pre-shock density, is set to 1 . The shock velocity ranges from the minimum value likely to generate sputtering of the grain cores (over $25 \mathrm{~km} \mathrm{~s}^{-1}$ ), to the maximum value permitted by the peculiar physics of those shocks and by the choice of such parameters (Flower et al. 2003). The age of the shock region can also be varied in our models, by varying their final flow time, that is the necessary time for a particle to flow from the pre-shock to the post-shock region. In practice, the final flow time is the time at which we stop the calculations.

The necessary outputs of the shock model, such as the temperature profile and fractional abundances of relevant species ( $\mathrm{SiO}$ and colliding species) are then used as inputs of the radiative-transfer code, which also makes use of $\mathrm{SiO}$ spectroscopic parameters (Einstein coefficients, collisional rate coefficients) from Dayou \& Balança (2006) and Schöier et al. (2005), and provided by the LAMDA database ${ }^{5}$, to solve the statistical equilibrium equations and calculate the level populations at each point of the shock. The set of equations is solved thanks to the escape probability formalism. The line emissivities and optical depth are also computed at each point of the shock layer for all considered transitions. Finally, we can compare the integrated intensity over the whole shock region, as resulting from the models, with the observations.

The 30' 5 beam of the APEX observations encompasses the blue- and red-lobe of OF1 (see Figs. 2 and 5, and also Fig. 6 for our definition of the velocity intervals associated with these lobes), which correspond to different shock regions detected at high velocities. It also comprises an "ambient velocity component" that we interpret as a consequence of the propagation of the shock wave. Indeed, this narrow, ambient-velocity component has already been seen in bipolar outflows associated to low-mass forming stars, and it could arise either from decelerating Si-enriched post-shock gas, mixed with the ambient gas (e.g., Lefloch et al. 1998; Codella et al. 1999), or from a slow reverse C-type shock brought to rest by the much denser ambient medium (Gusdorf et al. 2008a). In any case, this part of the $\mathrm{SiO}$ emission is not covered by our shock modelling, which solely describes the more dynamical $\mathrm{SiO}$ emission detected in the blue- and red-wings of our observations. Owing to its associated velocity, this $\mathrm{SiO}$ emission is interpreted as arising from a shock of outflow- or jet-like origin, a view that is also supported by the maps shown in Figs. 1, 2 and 5.

We hence compare the red-lobe and blue-lobe integrated intensities of the $\mathrm{SiO}(5-4)$ and (8-7) lines with the results generated by the grid of $\mathrm{C}$-shock models previously introduced in Gusdorf et al. (2008a), for pre-shock density values of $10^{4}, 10^{5}$, and $10^{6} \mathrm{~cm}^{-3}$. The observed integrated intensities are $2.1 \pm 0.4$ and $4.4 \pm 0.9 \mathrm{~K} \mathrm{~km} \mathrm{~s}^{-1}$ in the blue wings and $3.8 \pm 0.8$ and $6.7 \pm 1.3 \mathrm{~K} \mathrm{~km} \mathrm{~s}^{-1}$ in the red wings, for $\mathrm{SiO}(5-4)$ and (8-7) respectively. The velocity ranges used in the analysis are $[-30,-20] \mathrm{km} \mathrm{s}^{-1}$ for the blue-shifted emission, and $[+10,+39] \mathrm{km} \mathrm{s}^{-1}$ for the red-shifted emission as defined in Sect. 3.3 and labelled in Fig. 6. The error bars associated to these values correspond to the $20 \%$ calibration uncertainty intrinsic to our measurements.

From our SMA maps, we derived an observed area of $\sim 30 \operatorname{arcsec}^{2}$ for the $\mathrm{SiO}(5-4)$ emission in OF1, which corresponds to a deconvolved area of $\sim 22 \operatorname{arcsec}^{2}$, although the emission is unresolved along the minor axis of the emission. Therefore, we adopt this value as an upper limit to the size of the $\mathrm{SiO}$ emission associated with OF1. A second estimate of the

\footnotetext{
http://www.strw.leidenuniv.nl/ moldata/
}

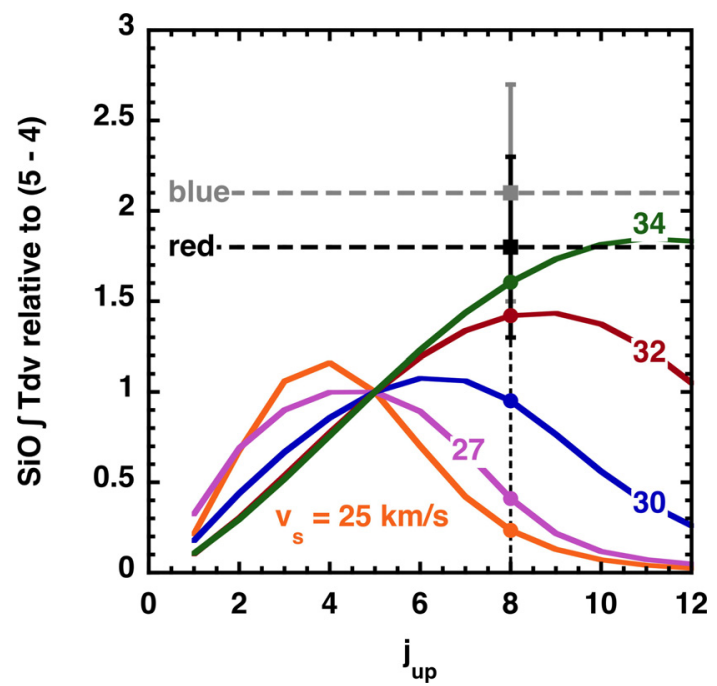

Fig. 8. Integrated intensities of the $\mathrm{SiO}$ lines relative to $\mathrm{SiO}(5-4)$. The horizontal lines show the values of the $\mathrm{SiO}(8-7)$ to $(5-4)$ ratio, whether it is the one in the blue or red lobe of the outflow. The vertical line marks the position of the (8-7) transition on the $X$-axis. The colour curves provide the results for the fraction of our C-type shock models grid relevant to our study. For all these models, the pre-shock density is $n_{\mathrm{H}}=$ $10^{6} \mathrm{~cm}^{-3}$, and the magnetic field parameter is $b=1$. Only the shock velocity $v_{\mathrm{s}}$ varies, and is indicated for each model on the corresponding line. The blue- and red-velocity range are defined in Fig. 6.

size of $\mathrm{OF} 1$ comes from the $\mathrm{H}_{2}$ emission. The assumption that $\mathrm{SiO}$ and $\mathrm{H}_{2}$ have similar sizes in protostellar outflows is justified by high-angular resolution observations of low-mass systems (e.g., Hirano et al. 2006; Codella et al. 2007), which clearly show that $\mathrm{SiO}$ knots have near-infrared $\mathrm{H}_{2}$ counterparts except at high extinctions associated with dense protostellar envelope where $\mathrm{H}_{2}$ is not detected. For the $\mathrm{H}_{2}$ counterpart of the red lobe of the $\mathrm{SiO}(5-4)$ emission shown in Fig. 1, we measure an emitting size of $\sim 6 \operatorname{arcsec}^{2}$. For the blue lobe, no $\mathrm{H}_{2}$ emission is detected, possibly owing to extinction. However, because OF1 is seen symmetric in both $\mathrm{SiO}$ and $\mathrm{SO}$ (Fig. 1), we assume an emitting size of $\sim 6 \operatorname{arcsec}^{2}$ for the blue-lobe, resulting in a total area $\sim 12 \operatorname{arcsec}^{2}$. Finally, we assume that the size of the emitting region is the same for both $\mathrm{SiO}$ lines; hence, the beam-filling factor is the same for both transitions, since the $\mathrm{SiO}(8-7)$ data were smoothed to the resolution of the $\mathrm{SiO}(5-4)$ observations (Sect. 2.1). This minimises possible biases due to beam dilution.

We base our selection of a "satisfyingly fitting model" on two criteria. First, we use the integrated intensity ratio of the $\mathrm{SiO}(8-7)$ to the $\mathrm{SiO}(5-4)$ lines. According to Fig. 8 (adapted from Fig. 9 of Gusdorf et al. 2008a), the only model reproducing the observations is that with $n_{\mathrm{H}}=10^{6} \mathrm{~cm}^{-3}, b=1$, and $v_{\mathrm{s}}=32 \mathrm{~km} \mathrm{~s}^{-1}$. Below this velocity, the sputtering and excitation conditions are not efficient enough, whereas above it, too much $\mathrm{SiO}(8-7)$ emission is generated to match the observations. Then, we check that the maximum line temperatures predicted by the model agree with the observed ones, which can be read in main beam units in Fig. 6: around $0.6 \mathrm{~K}$ in the red and blue wings, with a maximum slightly higher for $\mathrm{SiO}(8-7)$ than for $\mathrm{SiO}(5-4)$. Assuming that $\sim 60 \%$ of the APEX SiO emission originates in OF1 (Sect. 3.3), one must then account for a main beam maximum temperature of $\sim 0.36 \mathrm{~K}$. To convert this into brightness temperatures unit, we use both the upper limit of $22 \operatorname{arcsec}^{2}$ and the $\mathrm{H}_{2}$ emission size of $6 \operatorname{arcsec}^{2}$ for each lobe of $\mathrm{SiO}$ emission. The beam size of the APEX spectra being 30 '. 5 for both transitions, the $0.36 \mathrm{~K}$ main beam value amounts 
to $\sim 12$ and $\sim 44 \mathrm{~K}$ after correcting for dilution. This latter value agrees very decently with our models predictions (Fig. 8), where the line temperature profiles are displayed over the whole layer of the shock for the (5-4) and (8-7) transitions, showing maximum temperatures of respectively $49 \mathrm{~K}$ and $51 \mathrm{~K}$. We note that such a satisfactory agreement cannot be found for other models of our grid. This seems also to suggest an emitting size for the $\mathrm{SiO}(5-4)$ comparable to that of $\mathrm{H}_{2}, \sim 6 \operatorname{arcsec}^{2}$ per lobe.

We can then extract the surface density corresponding to this best model, and derive two values corresponding to 500 and 1000 years, which are the typical upper limits for the age of our shocks given in Paper I. We obtain 0.12 and $0.25 \mathrm{~g} \mathrm{~cm}^{-2}$. The second value is higher than the first one because an older shock age implies a more-extended post-shock region, where the gas is denser. We first derive the total outflow-mass comprised in the upper limit of our $\mathrm{SiO}$ emitting region size, $\sim 22 \mathrm{arcsec}^{2}$, adopting a distance of $1 \mathrm{kpc}$. We obtain a total mass of $0.3 M_{\odot}$ with the 500 years limit, and $0.6 M_{\odot}$ with the 1000 years limit. If we alternatively use the total emission region size of $\mathrm{H}_{2}$, namely $\sim 12 \operatorname{arcsec}^{2}$, we obtain a corresponding total outflowmass of $0.2 M_{\odot}$ for $500 \mathrm{yr}, 0.3 M_{\odot}$ for $1000 \mathrm{yr}$ approximately. The calculated masses agree with those inferred in Paper I $\left(0.6-0.9 M_{\odot}\right)$ and imply a luminosity of the driving source of OF1 of $\sim 10^{3} L_{\odot}$ based on the empirical outflow mass-luminosity relation of López-Sepulcre et al. (2009). This luminosity corresponds to that of a B3-type ZAMS star (e.g., Panagia 1973). However, our estimate of the total mass of the outflow has to be considered as a lower limit since only a limited range of velocities was used in the analysis. The inferred luminosity of the powering source of OF1 is also probably a lower limit because the outflow mass-luminosity relation of López-Sepulcre et al. (2009) is based on CO measurements.

\subsection{Slab-LVG analysis}

To verify these results and to constrain the excitation conditions of the OF1 SiO outflow, we also performed a "slab" analysis of the OF1 region using only an LVG code. We investigated the column density of $\mathrm{SiO}\left(N_{\mathrm{SiO}}\right)$ in the range $10^{11}-10^{12} \mathrm{~cm}^{-2}, \mathrm{H}_{2}$ densities $\left(n_{\mathrm{H}_{2}}\right)$ of $10^{3}-10^{8} \mathrm{~cm}^{-3}$ and kinetic temperatures $\left(T_{\text {kin }}\right)$ of 10-600 K. Figure 10 shows our typical model results for a kinetic temperature of 100 and $500 \mathrm{~K}$. We analysed the line intensities at LSR velocity as well as at the average velocities of the blue- $\left(-25 \mathrm{~km} \mathrm{~s}^{-1}\right)$ and red-shifted $\left(+15 \mathrm{~km} \mathrm{~s}^{-1}\right)$ lobes. Figure 10 indicates temperatures higher than $100 \mathrm{~K}$ for the red and blue lobes: lower temperatures would imply unreasonable densities higher than $10^{8} \mathrm{~cm}^{-3}$. The $n_{\mathrm{H}_{2}}$ values for $T_{\text {kin }}=500 \mathrm{~K}$ are $\simeq 5 \times 10^{6} \mathrm{~cm}^{-3}$. Since this is very likely an upper limit to the kinetic temperature of the gas, these densities have to be considered as lower limits. The inferred $\mathrm{SiO}$ column densities are $\sim 0.5-1 \times 10^{14} \mathrm{~cm}^{-2}$ for both lobes. On the other hand, at ambient velocity we can infer $N_{\mathrm{SiO}}$ of $2-5 \times 10^{13} \mathrm{~cm}^{-2}$ and densities higher than $10^{5} \mathrm{~cm}^{-3}$ without any clear constraint on kinetic temperature.

For consistency purposes, we cross-checked the results yielded by our two modelling methods, shock-LVG vs. slabLVG. To do so, one must return to the shock models and extract the ranges of variation over the shock layer of the parameters, which can only then be compared with the single-point values of the same parameters, which prevail in the slab-LVG analysis. The shock code outputs consist of a series of profiles of physical and chemical quantities (e.g., temperature, abundance) calculated over the shock layer. Our LVG code, when used in combination with the shock code, generates a series of outputs in

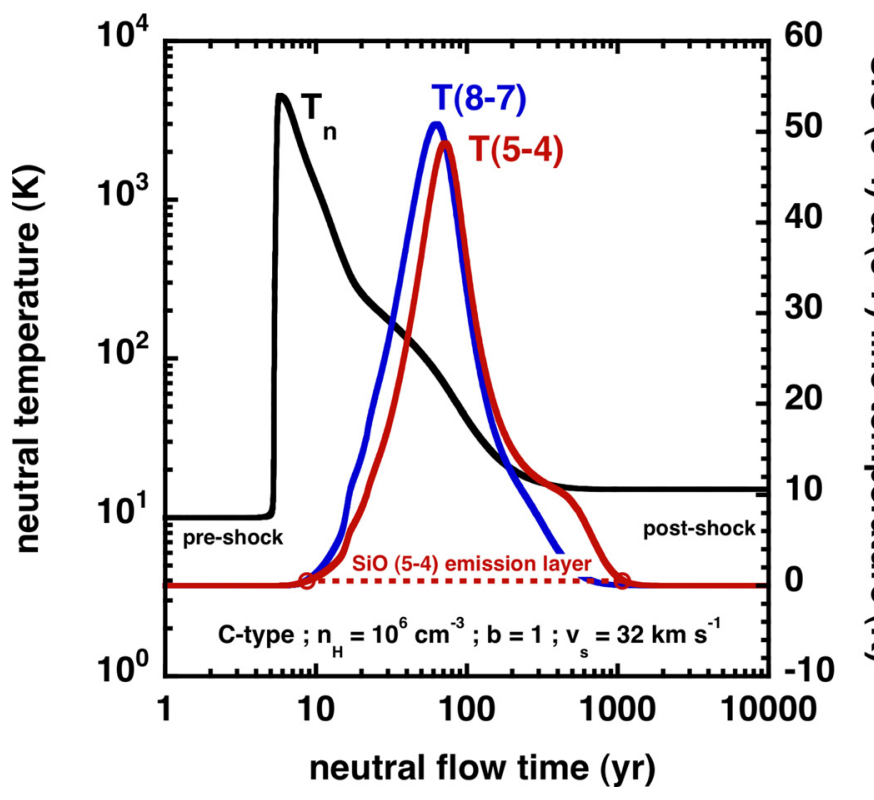

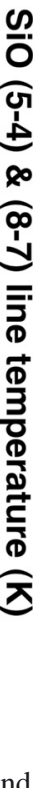

Fig. 9. Neutral temperature (black line) and the $\mathrm{SiO}(5-4)$ and (8-7) line temperature (red and blue lines) profiles over the whole shock layer, seen face-on, versus the neutral flow time for our best-fitting model. The characteristics of this model are indicated in the figure, as well as the location of pre- and post-shock regions. Our definition of the $\mathrm{SiO}(5-4)$ emission region (Sect. 4.2) is also delimited by the two red open circles and is represented by the horizontal dashed red line. In this diagram, the line of sight lies on the plane of the figure, in the direction that goes from the pre- to the post-shock region.

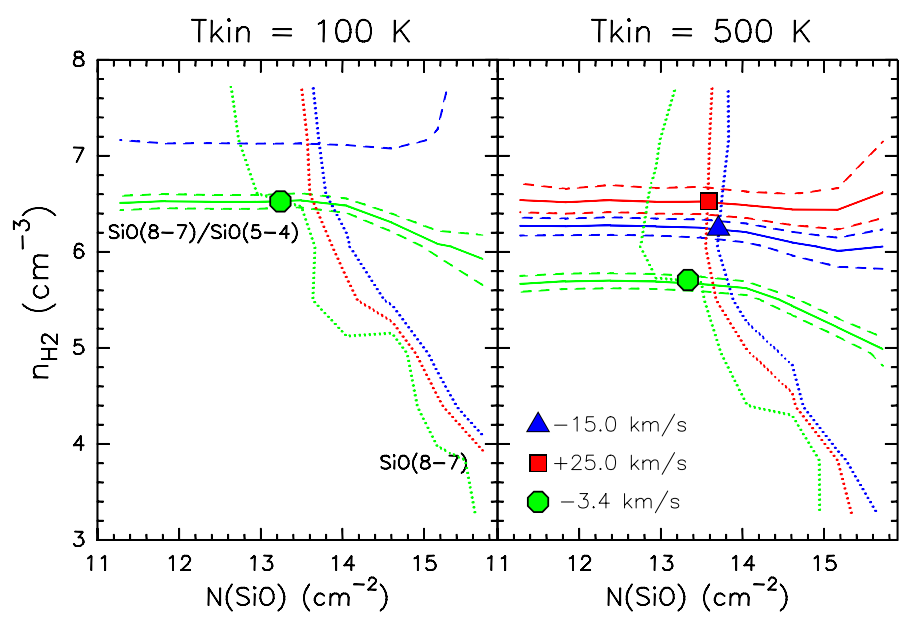

Fig. 10. Volume density $\mathrm{n}_{\mathrm{H}_{2}}$ versus $\mathrm{SiO}$ column density, $N(\mathrm{SiO})$ for slab-LVG models for $T_{\text {kin }}=100 \mathrm{~K}$ (left panel) and $500 \mathrm{~K}$ (right). Each continuous curve corresponds to the observed $\mathrm{SiO}(8-7) / \mathrm{SiO}(5-4)$ intensity ratio (after convolution to the same resolution, see Sect. 2.1), as measured at ambient velocity $\left(-3.4 \mathrm{~km} \mathrm{~s}^{-1}\right.$, green curve) and at typical red- $\left(+25.0 \mathrm{~km} \mathrm{~s}^{-1}\right.$, red curve $)$ and blue-shifted $\left(-15.0 \mathrm{~km} \mathrm{~s}^{-1}\right.$, blue curve) velocities. Dashed lines are for the ratio uncertainties. The dotted curves correspond to the APEX $\mathrm{SiO}(8-7)$ values at the same velocities, after correction for beam dilution using the OF1 size as derived from the SMA data. The corresponding uncertainties are negligible and are not shown for the sake of clarity. The symbols (circle: ambient; square: red; triangles: blue) indicate the allowed solutions.

the form of similar profiles of emissivities quantities (e.g., level populations, line emissivities). For the model that was chosen to describe the OF1 observations, Fig. 9 shows the neutral temperature and the local emissivity of the $\mathrm{SiO}(5-4)$ line over the whole shock layer, which is represented on the $x$-axis by means 
Table 3. Properties of SiO-emission regions inferred from face-on C-type shock models used in combination with an LVG code, and from homogeneous slab-LVG models.

\begin{tabular}{lcccc}
\hline \hline $\begin{array}{l}\text { Medium } \\
\text { properties }\end{array}$ & red lobe & $\begin{array}{c}\text { Slab-LVG } \\
\text { blue lobe }\end{array}$ & ambient & $\begin{array}{c}\text { Shock-LVG } \\
\text { best model }\end{array}$ \\
\hline$T_{\text {kin }}(\mathrm{K})$ & $100-500$ & $100-500$ & n.c. & $15-1745$ \\
$n_{\mathrm{H}_{2}}\left(\mathrm{~cm}^{-3}\right)$ & $\geq 5 \times 10^{6}$ & $\geq 5 \times 10^{6}$ & $\geq 10^{5}$ & $3.3 \times 10^{6}-2.4 \times 10^{7}$ \\
$N_{\mathrm{SiO}}\left(\mathrm{cm}^{-2}\right)$ & $5 \times 10^{13}$ & $10^{14}$ & $5 \times 10^{13}$ & $2.4 \times 10^{14}$ \\
LVG parameter & $2.5 \times 10^{12}$ & $5 \times 10^{12}$ & $2.5 \times 10^{12}$ & $6.4 \times 10^{11}-5.0 \times 10^{14}$ \\
\hline
\end{tabular}

of the neutral flow time of the shock, i.e. the necessary time for a neutral particle to fly through the shock region. This representation allows us to empirically define the $\mathrm{SiO}(5-4)$ emission region as the region where the emissivity of the line is significant. By significant we mean that the emission region consists of all the points where the emissivity of the line exceeds $1 \%$ of the maximum line temperature, that is about $0.5 \mathrm{~K}$ for the considered model. We then compare the range of variations over this emission region, of the quantities that are also used as input parameters in the slab-LVG method. This way, we performed a proper comparison between the range-values inferred from the shock-LVG analysis and the single-values yielded by the slabLVG method. These are reported in Table 3. In our shock-LVG models, only one parameter is not expressed in the form of a range of values: the $\mathrm{SiO}$ column density because it corresponds to the $\mathrm{SiO}$ density integrated over the whole shock layer. Note that the definition of the LVG parameter is the same in both the slab-LVG and shock-LVG cases, but that its expression differs. For the slab-LVG modelling, it is simply equal to $N_{\mathrm{SiO}}\left(\mathrm{cm}^{-2}\right) /$ $\Delta v\left(\mathrm{~km} \mathrm{~s}^{-1}\right)$, which is equivalent to the shock context formulation $10^{5} \times n(\mathrm{SiO})\left(\mathrm{cm}^{-3}\right) / \operatorname{grad} \_v\left(\mathrm{~s}^{-1}\right)$, where grad_v is the velocity gradient, and where the $10^{5}$ factor only arises because of the conversion from $\mathrm{km}$ to $\mathrm{cm}$.

The results shown in Table 3 are remarkably consistent and seem to support the view that tools that have been proved efficient in the context of low-mass star formation can be used in higher mass cases, with slightly increased densities. However, our best-fitting shock model was only approaching the observed $\mathrm{SiO}$ line ratios, and despite additional investigations in the parameter space, we were unable find a model likely to match $(8-7) /(5-4)$ ratio values as high as 2 , for instance. If confirmed as characteristic of massive star formation processes, such a high ratio could be accounted for by considering grain-grain interactions within the shock layer. Such interactions have been described as important at high pre-shock densities, and their feedback effects on both the chemical and dynamical structure of shocks have been studied by Guillet et al. (2007, 2009, 2011). Unfortunately, those authors could not generate any selfconsistent solution for the pre-shock densities at work here, namely for $n_{\mathrm{H}}=10^{6} \mathrm{~cm}^{-3}$. Alternatively, the influence of a strong radiation field generated by the driving source of the outflow both on the shock structure itself and on the corresponding molecular excitation might yield high $\mathrm{SiO}$ line ratios. The rather complex implementation of such processes in shock models is work in progress. The present analysis indicates volume densities higher than $10^{5} \mathrm{~cm}^{-3}$ (see Table 3). Nisini et al. (2007) performed an LVG analysis of $\mathrm{SiO}$ emission towards the nearby prototypical Class 0 low-mass objects L1148 and L1157, using single-dish data on angular scales of $10^{\prime \prime}$. No tight constraints have been obtained for kinetic temperature, whereas the volume densities were found to be between $10^{5}$ and $10^{6} \mathrm{~cm}^{-3}$. In addition, Gusdorf et al. (2008a) modelled the $\mathrm{SiO}$ emission from L1157 using a C-shock code leading to pre-shocked densities of $10^{4}-10^{5} \mathrm{~cm}^{-3}$. Thus, although completely different spa- tial scales are involved, the volume densities here inferred for IRAS 17233-3606 appear to be consistent with those derived for L1448 and L1157.

Although the results from the slab LVG approach are consistent with those inferred through the more rigorous shock-LVG analysis, we stress here the importance of shock models for deriving physical parameters such as the $\mathrm{SiO}$ abundance and therefore the mass of the outflow: while in the slab-LVG method one has to rely on assumed values of $X_{\mathrm{SiO}}, X_{\mathrm{SiO}}$ is consistently computed in the shock model, thus decreasing the uncertainties in the estimate of the outflow mass.

\section{The nature of OF1}

The present data sets trace the outflow activity driven by the YSOs in the cluster, and contribute to draw a clearer picture of the region than that obtained by our previous CO data (Paper I). Our previous observations of OF1 were limited to EHV redshifted $\mathrm{CO}(2-1)$ emission and to the detection of $\mathrm{H}_{2}$ emission also constrained to only the red lobe of OF1. The bipolar nature of OF1 was suggested only by the SO data and by water maser spots. Our present SMA data confirm that the emission associated with OF1 is due to a bipolar collimated outflow well traced by $\mathrm{SiO}$ in both red- and blue-shifted emission. This is consistent with findings for jets driven by Sun-like protostars, where it is possible to see that $\mathrm{CO}$ traces not only the jet but also the walls of the cavity opened by the jet itself (e.g., Lee et al. 2007, their Fig. 5; and Cabrit et al. 2012, their Fig. 1). On the other hand, $\mathrm{SiO}$ emission is usually associated with primary jets in the case of low-mass YSOs (e.g., Hirano et al. 2006; Cabrit et al. 2012) and generally suffers minimal contamination from infalling envelopes or swept-up cavities.

Our data strongly suggest that the emission associated with OF1 traces a bipolar jet from the IRAS 17233-3606 cluster. This view is additionally supported by the high velocities detected in $\mathrm{SiO}$, and by the collimated morphology of OF1, which is $\sim 6$ according to the $\mathrm{H}_{2}$ images, among the largest ever reported in high-mass star formation (see for example Beuther et al. 2002, 2004; Su et al. 2004; Wang et al. 2011). This value corresponds to the ratio between the major semi-axis and the minor semiaxis of $\mathrm{OF} 1$ as derived from the $\mathrm{H}_{2}$ map. Additionally, the high temperature $(T>100 \mathrm{~K})$ derived through the $\mathrm{SiO}$ analysis is suggestive of material very close to the primary jet.

Although the driving source of OF1 cannot be identified yet given the still relatively low resolution of the SMA data ( $\sim 3000 \mathrm{AU})$, the best candidate source is VLA-2a, which is the strongest $\mathrm{cm}$-continuum source in the region and also the compact source closest to the $\mathrm{H}_{2}$ jet-like feature. Alternatively, VLA-2b could also be a possible candidate for the powering source of OF1. In principle, we cannot rule out the hypothesis that the driving source of the observed $\mathrm{SiO}$ bipolar emission is a low-mass YSO in the cluster. However, there is some indirect evidence that OF1 is indeed driven by a massive YSO. First, we determined the total mass of OF1 through the shock modelling 
of $\mathrm{SiO}$ emission without any assumption of the $\mathrm{SiO}$ abundance (Sect. 4.1) and using the area inferred from the $\mathrm{H}_{2}$ emission. From this mass, we inferred a luminosity of $\geq 10^{3} L_{\odot}$ for the OF1 driving source, which agrees with an earlier than B3-type star. The continuum flux of VLA-2a in the $\mathrm{cm}$ (Zapata et al. 2008) is also compatible with that of an early B ZAMS star. The cm continuum flux of VLA-2b (Zapata et al. 2008) is also compatible with an early B-type star, although less massive than VLA-2a (a B3 ZAMS star compared to a B1 ZAMS star). To our knowledge, this is one of the first indirect evidences of a $\mathrm{SiO}$ collimated bipolar outflow from an early B type YSO, although only higher spatial resolution maps will allow a direct proof of its existence. Previous studies reported red- and blue-shifted $\mathrm{SiO}$ emission from massive star-forming regions (Cesaroni et al. 1999; Hunter et al. 1999; Qiu et al. 2007; Zhang et al. 2007; Codella et al. 2013). Among these, IRAS 20126+4104 (e.g., Cesaroni et al. 1999; Caratti o Garatti et al. 2008) probably represents the best example of a jet from a massive YSO, since the nature of the jet is proved by $\mathrm{SiO}, \mathrm{H}_{2}$ and [FeII] observations. However, the mass of the powering source is $\sim 7 M_{\odot}$ (Cesaroni et al. 2005). In one case (IRAS 18264-1152, Qiu et al. 2007) SiO is also detected at high velocities, as in the present case of OF1. However, the difference between these studies and our case is that several features typical of low-mass jets (collimation, emission at high and extremely high velocity, the parameters of the gas derived by the shock model) are all found in OF1 while the other examples show only some of these properties.

\section{Conclusions}

We reported and analysed SMA and APEX SiO observations of the IRAS 17233-3606 star-forming region. The SMA data allowed us to clearly resolve the OF1 outflow with a resolution of $\sim 3000 \mathrm{AU}$ and identify its $\mathrm{SiO}$ emission as due to a collimated bipolar outflow associated with EHV $\mathrm{CO}, \mathrm{H}_{2}$ and $\mathrm{SO}$.

The OF1 SiO(5-4) and (8-7) APEX spectra suggest an increase of the excitation conditions at high velocities and point to hot gas close to the primary jet, as found in outflows from lowmass YSOs. From the shock-LVG analysis of the APEX spectra, we derived a mass of $>0.3 M_{\odot}$ for $\mathrm{OF} 1$, which implies a luminosity $L \geq 10^{3} L_{\odot}$ for the OF1 driving source. Although the driving source of OF1 cannot be yet identified at the resolution of our current SMA data, the best candidate source, VLA-2a, has a continuum flux in the $\mathrm{cm}$ that also agrees with that of a ZAMS star with a luminosity of a few $10^{3} L_{\odot}$.

The high angular resolution spectroscopic observations that we collected on OF1 in IRAS 17233-3606 support a picture similar to that seen in studies of jets and outflows from low-mass YSOs. The $\mathrm{SiO}$ emission at LV peaks closer to the driving source than the $\mathrm{SiO}-\mathrm{HV}$ peak; moreover, $\mathrm{SiO}$ traces a region of the outflow/jet system heavily obscured in the $\mathrm{H}_{2}$ image. Finally, the $\mathrm{SiO}$ and SO molecules have a very similar distribution as found in collimated outflows driven by low-mass Class 0 YSOs.

In terms of shock modelling, we found it possible to model $\mathrm{SiO}$ emission in shock regions generated by high-mass protostars with the same tools developed for shocks from low-mass YSOs. The shock parameters inferred by the SiO modelling are compatible with those found for low-mass protostars, only with higher pre-shock densities. The same conclusions arise from a simpler LVG slab analysis.

Acknowledgements. The authors would like to thank the anonymous referee who helped to clarify the results and improve the discussion. A. Gusdorf acknowledges support by the grant ANR-09-BLAN-0231-01 from the French Agence Nationale de la Recherche as part of the SCHISM project.

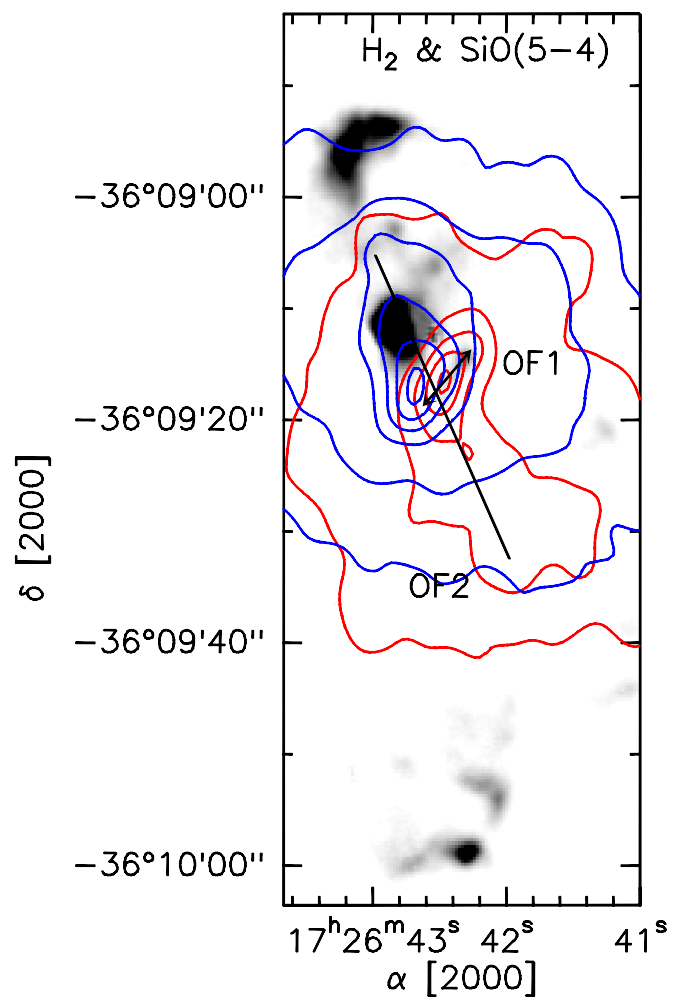

Fig. A.1. Integrated emission of the $\mathrm{SiO}(5-4)$ line in the SMA+APEX combined data. The blue and red contours are the blue- and red-shifted emission $\left(v_{\mathrm{bl}}=[-50,-20] \mathrm{km} \mathrm{s}^{-1}\right.$, from $10 \sigma(1 \mathrm{Jy} /$ beam $)$ in steps of $10 \sigma ; v_{\mathrm{rd}}=[+10,+39] \mathrm{km} \mathrm{s}^{-1}$, from $10 \sigma(1 \mathrm{Jy} /$ beam $)$ in steps of $10 \sigma)$. The grey image represents the $\mathrm{H}_{2}$ emission at $2.12 \mu \mathrm{m}$.

\section{Appendix A: Joint deconvolution of the SMA and APEX data}

We performed a joint deconvolution of the SMA and APEX observations in the image plane using the task feather in the Common Astronomy Software Applications (CASA, McMullin et al. 2007). A snapshot of the results of joint deconvolution of SMA and APEX data is shown in Fig. A.1. While the large-scale details are filtered out by the interferometer, the combined image restores the large-scale information. i.e. (1) the combined image faithfully reproduces the large-scale (extended) structure, (2) the joint deconvolution image also conserved the total flux as observed with the single-dish. We tested this by smoothing the combined image to the resolution of the single dish beam. We also attempted to combine the data sets in the UV plane. However, the flux calibration factor (ratio of single-dish to interferometer flux in the overlapping UV space) was unexpectedly high. Although we could not ascertain the reason for the large scaling factor, the smaller area mapped with the single dish relative to SMA area might contribute to the failed combination.

In Sect. 3.3, we demonstrated that the SMA recovers the whole flux observed with APEX in the velocity ranges used in the analysis in Sect. 4 and therefore the combination of the two datasets was not needed for our purposes.

\section{References}

Beuther, H., Schilke, P., Sridharan, T. K., et al. 2002, A\&A, 383, 892 Beuther, H., Schilke, P., \& Gueth, F. 2004, ApJ, 608, 330

Bonnell, I. A., Larson, R. B., \& Zinnecker, H. 2007, Protostars and Planets V, 149

Bronfman, L., Nyman, L.-A., \& May, J. 1996, A\&AS, 115, 81 
Cabrit, S., Codella, C., Gueth, F, et al. 2007, A\&A, 468, L29

Cabrit, S., Codella, C., Gueth, F., \& Gusdorf, A. 2012, A\&A, 548, L2

Caratti o Garatti, A., Froebrich, D., Eislöffel, J., Giannini, T., \& Nisini, B. 2008, A\&A, 485, 137

Caselli, P., Hartquist, T. W., \& Havnes, O. 1997, A\&A, 322, 296

Cesaroni, R., Felli, M., Jenness, T., et al. 1999, A\&A, 345, 949

Cesaroni, R., Neri, R., Olmi, L., et al. 2005, A\&A, 434, 1039

Codella, C., Bachiller, R., \& Reipurth, B. 1999, A\&A, 343, 585

Codella, C., Cabrit, S., Gueth, F., et al. 2007, A\&A, 462, L53

Codella, C., Beltrán, M. T., Cesaroni, R., et al. 2013, A\&A, 550, A81

Dayou, F., \& Balança, C. 2006, A\&A, 459, 297

Flower, D. R., \& Pineau des Forêts, G. 2003, MNRAS, 343, 390

Flower, D. R., Le Bourlot, J., Pineau des Forêts, G., \& Cabrit, S. 2003, MNRAS, 341,70

Guillet, V., Pineau des Forêts, G., \& Jones, A. P. 2007, A\&A, 476, 263

Guillet, V., Jones, A. P., \& Pineau des Forêts, G. 2009, A\&A, 497, 145

Guillet, V., Pineau Des Forêts, G., \& Jones, A. P. 2011, A\&A, 527, A123

Gusdorf, A., Cabrit, S., Flower, D. R., \& Pineau des Forêts, G. 2008a, A\&A, 482,809

Gusdorf, A., Pineau des Forêts, G., Cabrit, S., \& Flower, D. R. 2008b, A\&A, 490, 695

Gusdorf, A., Giannini, T., Flower, D. R., et al. 2011, A\&A, 532, A53

Hirano, N., Liu, S.-Y., Shang, H., et al. 2006, ApJ, 636, L141

Hollenbach, D. 1997, in Herbig-Haro Flows and the Birth of Stars, eds. B. Reipurth, \& C. Bertout, IAU Symp., 182, 181

Hunter, T. R., Testi, L., Zhang, Q., \& Sridharan, T. K. 1999, AJ, 118, 477

Klein, B., Philipp, S. D., Krämer, I., et al. 2006, A\&A, 454, L29

Lada, C. J., \& Fich, M. 1996, ApJ, 459, 638

Le Bourlot, J., Pineau des Forêts, G., Flower, D. R., \& Cabrit, S. 2002, MNRAS, 332,985

Le Picard, S. D., Canosa, A., Pineau des Forêts, G., Rebrion-Rowe, C., \& Rowe, B. R. 2001, A\&A, 372, 1064
Lee, C.-F., Ho, P. T. P., Palau, A., et al. 2007, ApJ, 670, 1188

Lefloch, B., Castets, A., Cernicharo, J., \& Loinard, L. 1998, ApJ, 504, L109

Leurini, S., Hieret, C., Thorwirth, S., et al. 2008, A\&A, 485, 167

Leurini, S., Codella, C., Zapata, L. A., et al. 2009, A\&A, 507, 1443

Leurini, S., Codella, C., Zapata, L., et al. 2011a, A\&A, 530, A12

Leurini, S., Pillai, T., Stanke, T., et al. 2011b, A\&A, 533, A85

López-Sepulcre, A., Codella, C., Cesaroni, R., Marcelino, N., \& Walmsley, C. M. 2009, A\&A, 499, 811

May, P. W., Pineau des Forêts, G., Flower, D. R., et al. 2000, MNRAS, 318, 809

McKee, C. F., \& Tan, J. C. 2002, Nature, 416, 59

McKee, C. F., \& Tan, J. C. 2003, ApJ, 585, 850

McMullin, J. P., Waters, B., Schiebel, D., Young, W., \& Golap, K. 2007, in Astronomical Data Analysis Software and Systems XVI, eds. R. A. Shaw, F. Hill, \& D. J. Bell, ASP Conf. Ser., 376, 127

Nisini, B., Codella, C., Giannini, T., et al. 2007, A\&A, 462, 163

Panagia, N. 1973, AJ, 78, 929

Qiu, K., Zhang, Q., Beuther, H., \& Yang, J. 2007, ApJ, 654, 361

Sault, R. J., Teuben, P. J., \& Wright, M. C. H. 1995, in Astronomical Data Analysis Software and Systems IV, ASP Conf. Ser., 77, 433

Schilke, P., Walmsley, C. M., Pineau des Forets, G., \& Flower, D. R. 1997, A\&A, 321,293

Schöier, F. L., van der Tak, F. F. S., van Dishoeck, E. F., \& Black, J. H. 2005, A\&A, 432, 369

Su, Y.-N., Zhang, Q., \& Lim, J. 2004, ApJ, 604, 258

Tafalla, M., Santiago-García, J., Hacar, A., \& Bachiller, R. 2010, A\&A, 522, A91

Wang, K, Zhang, Q., Wu, Y, \& Zhang, H. 2011, ApJ, 735, 64

Wrathmall, S. A., Gusdorf, A., \& Flower, D. R. 2007, MNRAS, 382, 133

Zhang, Q., Sridharan, T. K., Hunter, T. R., et al. 2007, A\&A, 470, 269

Zapata, L. A., Leurini, S., Menten, K. M., et al. 2008, AJ, 136, 1455

Zapata, L. A., Ho, P. T. P., Schilke, P., et al. 2009, ApJ, 698, 1422 